\title{
Synthesis Of Zincoxide Nanorods And Its Application To Humidity Sensor
}

\author{
Indra Bahadur Karki ${ }^{1}$, Jeevan Jyoti Nakarmi ${ }^{2}$ and Suman Chatterjee ${ }^{3}$ \\ ${ }^{1}$ Patan Multiple Campus, Department of Physics, Tribhuvan University, Kathmandu \\ ${ }^{2}$ Central Department of Physics, Tribhuvan University, Kathmandu \\ ${ }^{3}$ Department of Physics, University of North Bengal, Siliguri- 734013, India \\ Email:-indrakarky@gmail.com
}

\begin{abstract}
The present study is concerned the sensing behavior of $\mathrm{ZnO}$ nanorods thin films application to humidity sensors. $\mathrm{ZnO}$ nanorods were fabricated on a glass substrate by sol-gel spin coating technique. The crystal structure of the resulting thin films was investigated by X-ray diffraction (XRD). Minimum crystalline grain size of this film was found about $5 \mathrm{~nm}$. Surface morphology of the film was investigated by Scanning Electron Microscope (SEM). SEM image shows rods like films which are evenly distributed on the surface of the sample. The film on a glass substrate explored for humidity sensing of controlled humid environment by measuring electrical resistance. The resistance falls from $\mathrm{M} \Omega$ to $\mathrm{K} \Omega$ as percentage relative humidity increases from 20 to $90 \%$.
\end{abstract}

Keywords: Zinc oxide nanorod, thinfilm, sensor, XRD, SEM

\section{INTRODUCTION}

Nanostructured semiconductor metal oxides gas sensors have attracted the attention of many researchers due to their exceptional properties (Erol et al. 2011). The preference of zinc oxide has gained importance because it synthesizes into nanostructures easily. In recent years, nanomaterials have attracted considerable attention for application in humidity sensors due to their sensitivity to humidity variation caused by their superior surface to volume ratio as compared to bulk materials (Erol et al 2011).

Sensors are electrical or mechanical components that are used to measure a property or behavior of an object or system. It is a device that responds to a physical stimulus such as heat, light, sound, pressure, magnetism or motion (Sun 2009).

Humidity sensors are gaining importance in everyday life. Humidity sensors are useful for the detection of the relative humidity in various environments. Humidity sensors are becoming necessary element in various fields such as textile industry, food processing, and building etc. (Sun 2009). High and low humidity affects human beings adversely. Excessive high humidity causes corrosion in metallic components in humid regions. Therefore, humidity is an important measure in the control of electronic goods production. Recently, there have been increased demands for humidity sensing elements for use in automatic humidity controls systems. Modern humidity sensing device is shown in Fig.1.

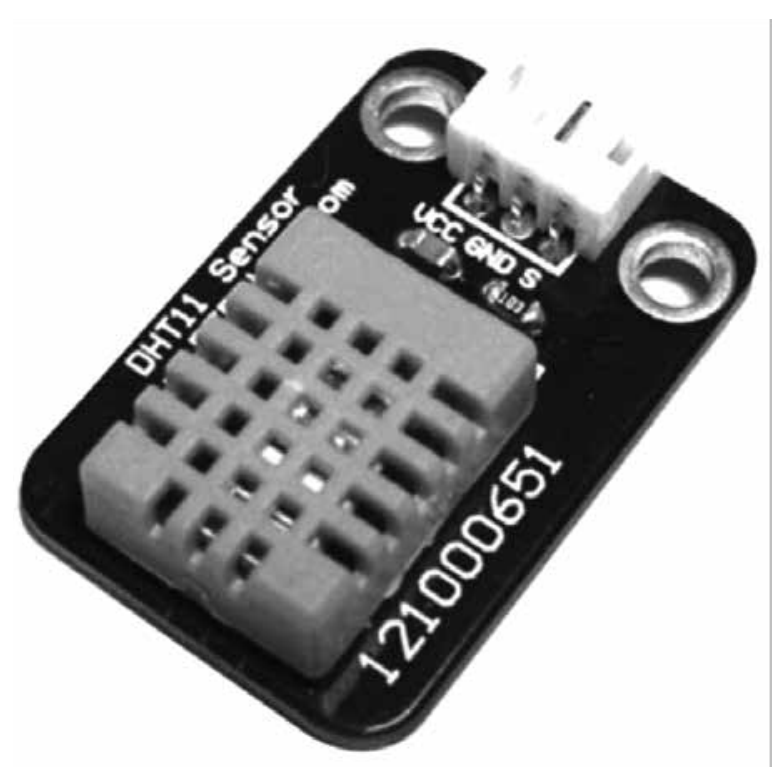

Fig. 1. Automatic Modern humidity sensing device.

Humidity sensors today are mostly of polymers and ceramics. Many metal oxides such as $\mathrm{SnO}_{2}, \mathrm{TiO}_{2}, \mathrm{Cr}_{2} \mathrm{O}_{3}$ and $\mathrm{WO}_{3}$ have been investigated as humidity sensors (Gao 2005). 
In recent years, the synthesis of nanocrystalline oxide materials has been a focal point of research and development acivities in the area of nanomaterials owing to the quest for their various technological applications (Srivastava 2006, Chatterjee 1999). Zinc oxide (ZnO) is an inorganic, semiconducting material that shares its structure with $\mathrm{ZnS}$ (wurtzite). $\mathrm{ZnO}$ nanoparticles have attracted interest in part because of their optical properties. These particles are transparent to visible light, making them invisible to the eye, but they absorb ultraviolet light. One application of this material is therefore in UV-blocking coatings, including cosmetics and sunscreens (Chatterjee 1999).

The objective of the present study is to synthesize $\mathrm{ZnO}$ nanorod thin film using sol-gel spin coating technique and to investigate humidity sensitive electrical properties of the pure $\mathrm{ZnO}$ nanorod sensing elements using d.c. measurements.

Humidity is most often measured by relative humidity, which is the ratio of moisture in the air to the maximum possible saturation of air at a given temperature. The warmer the air is, the more water vapor it can hold. Absolute humidity is the actual amount of water molecules in the air. The Schematic diagram for humidity measuring set up is shown in Fig. 2.

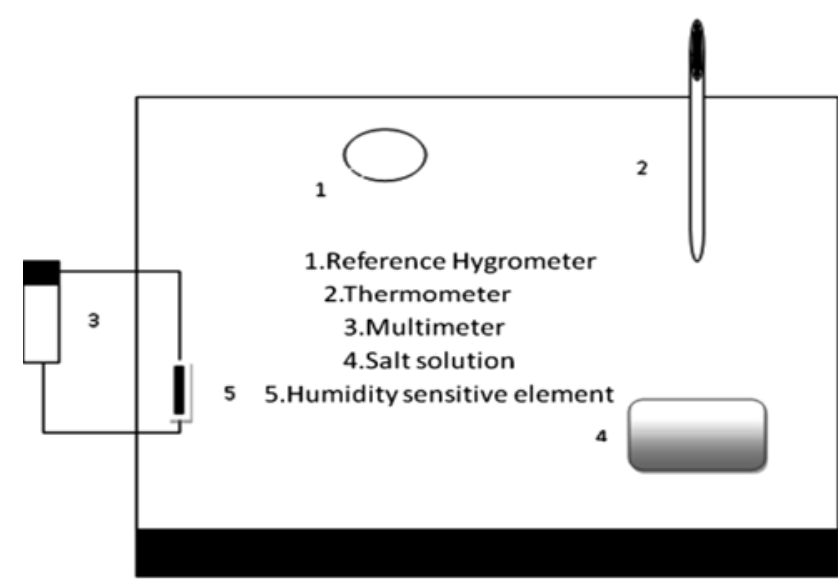

Fig 2. Schematic diagram for humidity measuring set up.

\section{Mechanism of Semiconducting Sensing Materials}

Some metal based composite oxides such as $\mathrm{ZnO}, \mathrm{SnO}_{2}$, and $\operatorname{In}_{2} \mathrm{O}_{3}$, etc. are wide-bandgap semiconductors. $\mathrm{H}_{2} \mathrm{O}$ is adsorbed on the oxide surface in molecular and hydroxyl forms. Water molecules are observed to increase the conductivity of n-type ceramics and to decrease the conductivity of p-type ceramics (Boyle 1997, Avani et al. 1981). This effect has been attributed to the donation of electrons from the chemically adsorbed water molecules to the ceramic surface (Boyle 1977). Another mechanism was proposed by (Yamazoe et al. 1979, Shimizu 1989). It was suggested that water molecules replace the previously adsorbed and ionized oxygen $\left(\mathrm{O}^{-}, \mathrm{O}^{2-}\right.$, etc.) and therefore release the electrons from the ionized oxygen (Shimizu 1989, Mukode 1989). Probably the "donor effect" could be resulted from both.

Because the conductivity is caused by the surface concentration of electrons, this sensing style is usually called "electronic type." However, the water layer formed by the physical adsorption may be somewhat protonconductive. Therefore, at room temperatures the conductivity of ceramic semiconducting materials is actually due to addition of both electrons and protons (ionic), unless at high temperatures $\left(>100{ }^{\circ} \mathrm{C}\right)$ moisture cannot effectively condense on the surface. In Fig.3a, the conductivity increment is produced by surface electron accumulation resulting from the preferential alignment of the water dipoles (Yamazoe et al. 1979). Hydrogen atoms contact the surface (mostly at the oxygen sites) and attract electrons outward (Fig. 3). In Fig. 3b, a depletion region forms originally due to adsorbed oxygen and the released electrons may neutralize the depletion. Since adsorbed water molecules increase the conductivity of n-type ceramic semiconductors, nearly all the published works deal with n-type ceramics. It was reported that the change of conductivity was linear to certain exponential based on the proposed surface reaction mechanism (Shimizu, 1989). However, most of works lack the derivation for establishing strict reaction models (Mukode 1989).

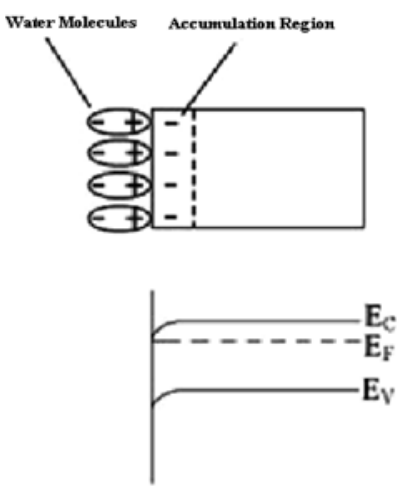

(a)
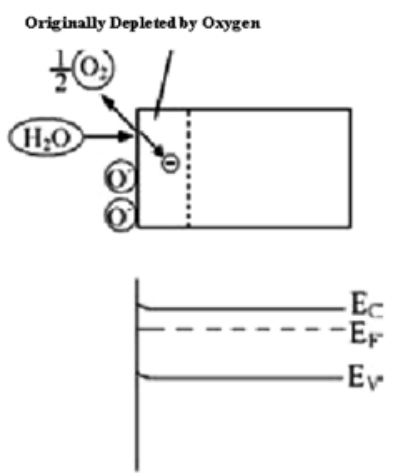

(b)
Fig. 3. Two possible mechanisms for the "donor effect" (just for n-type): (a) Electrons are attracted by the adsorbed water molecules to the semiconductor surface and the energy bands are bended; (b) Electrons are released by the competitive adsorption (Chen 2005). 


\section{MATERIALS AND METHODS}

\section{Synthesis of $\mathrm{ZnO}$ nanorod thinfilms}

$\mathrm{ZnO}$ nanorods thin films were prepared by spin coating sol-gel technique (Pradhan et al. 2007 and Karki et al. 2013). $\mathrm{ZnO}$ nanoseed were spin-coated using zinc acetate solution In this process, we prepared $5 \mathrm{mM}$ solution of zinc acetate dehydrate, $\left(\mathrm{CH}_{3} \mathrm{COO}\right)_{2} \mathrm{Zn}, 2 \mathrm{H}_{2} \mathrm{O}$, (98\% Merck) with distilled methanol. The solution was spun on soda-lime glass substrate $\left(2 \times 2 \mathrm{~cm}^{2}\right)$ at 1000 rpm for 30s using a Programmable Spin Coater (Apex Technologies, Model SCU-2008C). The substrates, now covered with a film of zinc acetate crystallites, were heated to $350{ }^{\circ} \mathrm{C}$ in an conventional oven for $30 \mathrm{~min}$ to yield layers of $\mathrm{ZnO}$ islands with their (100) plane parallel to the substrate surface. After evaporation of solvent, a thin $\mathrm{ZnO}$ nanorods film ware formed.

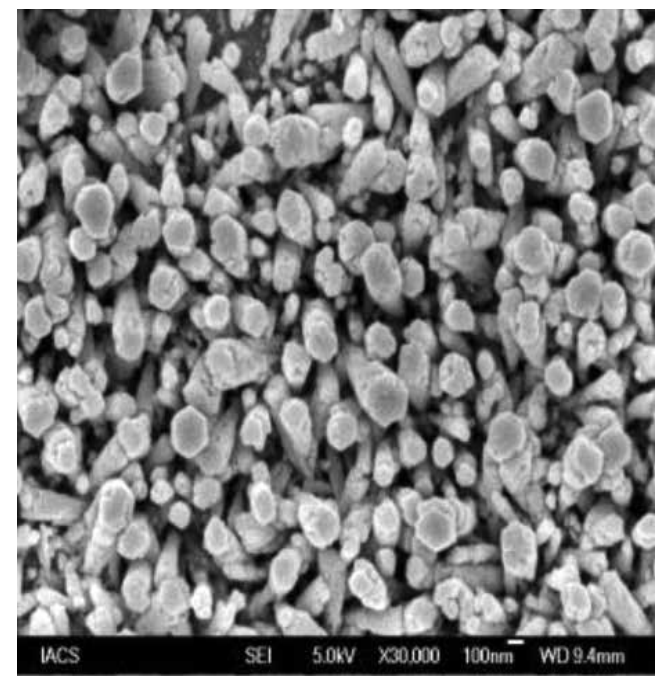

\section{RESULTS AND DISCUSSION}

\section{Characterizations of synthesized material}

\section{X-Ray Diffraction}

The crystal structure of $\mathrm{ZnO}$ nanorod thinfilms were analyzed using X-ray Difractometer.XRD pattern of the synthesized sensing materials shown in Fig. 4 reveals the crystalline nature of samples. The average crystallite size of the sensing material was calculated by Debye-Scherer formula, which is as follows:

$$
\mathbf{D}=\mathbf{K} \lambda / \boldsymbol{\beta} \cos \theta
$$

where $\mathrm{D}$ is the average size of crystallite and a fixed number $\mathrm{K}=0.94, \lambda$ the wavelength of $\mathrm{X}$-ray radiation having value $1.5418 \AA, \beta$ the full width at half maximum (FWHM) of the diffraction peak and $\theta$ the angle of diffraction.

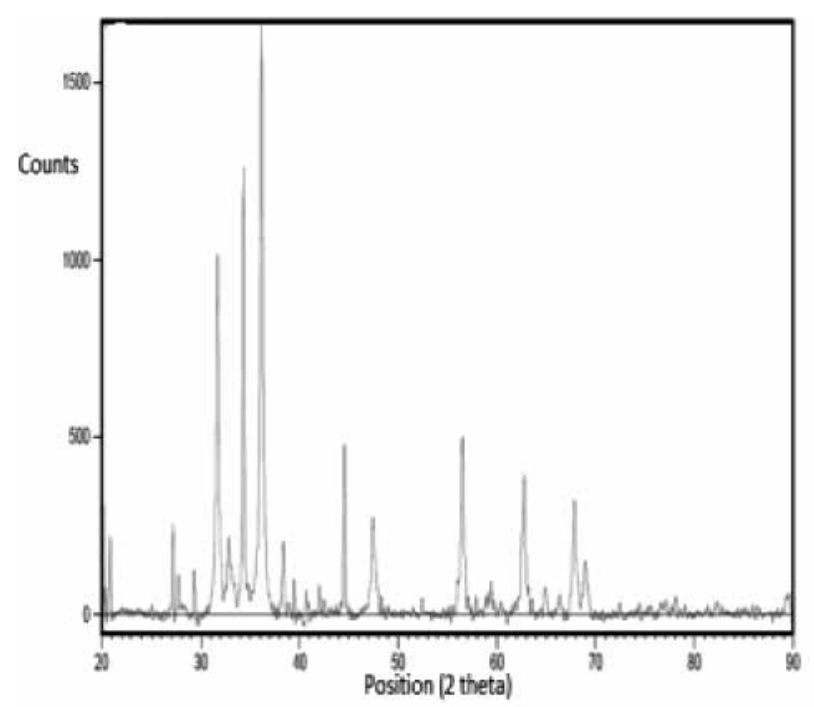

Fig. 4. SEM images of $\mathrm{ZnO}$ nanorods with hexagonal shapes and XRD pattern of $\mathrm{ZnO}$ films.

The nanorods have an average length of $100 \mathrm{~nm}$, diameter ranging from 10 to $20 \mathrm{~nm}$ and they are mostly vertically aligned with the substrate.

\section{Surface morphology}

Surface morphology of sensing element was studied using scanning electron microscope (SEM). Scanning electron micrographs of the sensing element annealed at $350^{\circ} \mathrm{C}$ are shown in Fig. 4 on microscale. SEM shows that crystallites of $\mathrm{ZnO}$ combining with adhesive glass particles beautiful form hexagonal shape and clusters, leaving more spaces as pores.

Higher porosity increases the surface-to-volume ratio of the materials, which in turn increases the diffusion rate of gas and therefore helps in getting good sensitivity. SEM shows that the crystallites are rod types, uniform in hexagonal shape and equally distributed.

The semiconducting humidity sensors usually work utilizing the mechanism of chemical and physical adsorption of water vapour on the surface of the material in a quantity. It is proportional to the $\% \mathrm{RH}$ of the surroundings. The adsorption generates the variation in the electrical resistance with \%RH. Fig. 5 shows the resistance varies with the relative humidity $(\mathrm{RH})$ in the range of $20-90 \%$ at $25{ }^{\circ} \mathrm{C}$. It can be seen in the device that the resistance drops rapidly as the humidity increases.

Fig. 5 also shows humidity sensing characteristics of $\mathrm{ZnO}$ thinfilm and there is linear decrease in electrical resistance up to higher range of $\% \mathrm{RH}$. This linear change in resistance is most significant for device fabrication. 


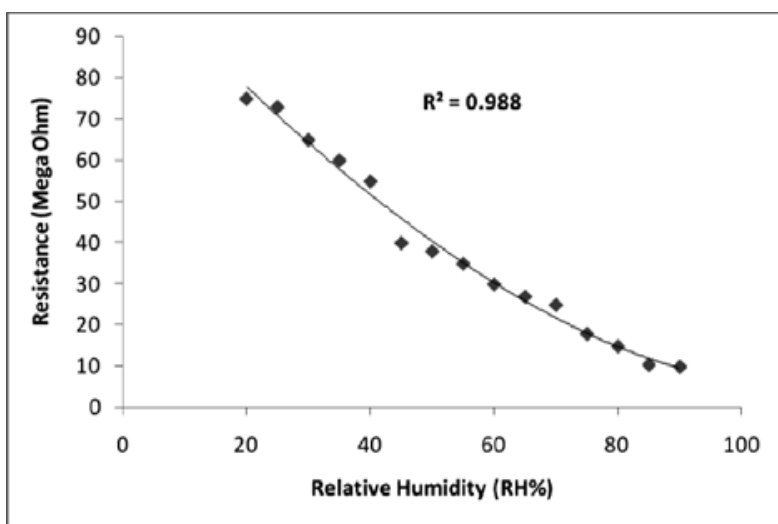

Fig. 5. Variation in resistance with percentage of $R H$.

Sensitivity of a humidity sensor can be defined as the change in resistance $(\mathrm{R})$ of sensing element per unit change in relative humidity ( $\% \mathrm{RH})$, is given by:

$$
\mathbf{S}=\Delta \mathbf{R} / \Delta \mathbf{R H} \% \quad \mathrm{M} \Omega / \% \mathrm{RH}
$$

The average sensitivity is calculated by taking the average of all sensitivities ranging from 20 to $90 \% \mathrm{RH}$. The average sensitivity of the thin film is $2.8 \mathrm{M} \Omega / \% \mathrm{RH}$. It has been observed during the experiment that as annealing temperature increases average sensitivity increases. In our experiment, the sensing film shows more adsorption sites; therefore it is sensitive to humidity. The correlation coefficient $\left(\mathbf{R}^{2}\right)$ of the plot of resistance versus $\mathrm{RH}$ is high which suggests that the electrical resistance varies with the relative humidity could be best described. The plot of resistance versus RH from $20 \%$ to $90 \%$, illustrates good reproducibility and excellent humidity sensitivity as shown in Fig. 5.

\section{CONCLUSION}

$\mathrm{ZnO}$ nanorods thin film on glass substrate were successfully synthesized using spin coating sol-gel method. Surface morphology of the $\mathrm{ZnO}$ nanorod films shows particles are almost hexagonal in shape and form clusters leaving more space as the pores. The morphological changes, as revealed by SEM, also contribute towards increase in sensitivity. The value of sensitivity was found $2.8 \mathrm{M} \Omega / \% \mathrm{RH}$. The resistance falls from $\mathrm{M} \Omega$ to $\mathrm{K} \Omega$ as percentage relative humidity increases from 20 to $90 \%$. This type of sensor is easy to fabricate and cheap. These experimental results show that the $\mathrm{ZnO}$ based sensor have satisfactory sensitivity and selectivity over the entire range of humidity.

\section{ACKNOWLEDGEMENTS}

The authors would like to thank the North Bengal University, Darjeeling, Siliguri for providing necessary laboratory facilities for the experimental work. Mr. I.B. Karki thankful to the University Grants Commission (UGC), Nepal for the PhD fellowship.

\section{REFERENCES}

Avani G.N. and Nanis, L. 1981. Effects of humidity on hydrogen-sulfide detection by $\mathrm{SnO}_{2}$ solid-state gas sensors. Sensors Actuators B 2: 201-206.

Boyle J.F. and Jones K.A. 1977. The effects of CO, water vapor and surface temperature on the conductivity of $\mathrm{SnO}_{2}$ gas sensor. Journal Electronic Materials 6: 717-733.

Chatterzee A.P., Mitra, P., Mukhopadhyay A.K. 1999. Chemically deposited zinc oxide thin film gas sensor. Journal of Materials Science 34(17): 4225-4231.

Chen Z. and Lu C. 2005. Humidity Sensors: A Review of Materials and Mechanisms. Sensor Letters 3: 274-295.

Erol A., Okur S., Yağmurcukardes N. and Arıkan M.C. 2011. Humidity-sensing properties of a $\mathrm{ZnO}$ nanowire film as measured with a QCM. Sensors and Actuators B: Chemical 152(1): 115-120.

Gao T., Wang T.H. 2005. Synthesis and properties of multipod-shaped $\mathrm{ZnO}$ nanorods for gas-sensor applications, Applied Physics A 80: 1451-1454.

Geng W., Li N., Li X., Wang R., Tu J., and Zhang T. 2007. Effect of polymerization time on the humidity sensing properties of polypyrrole. Sensors and Actuators B. 125: 114-119.

Karki I.B., Nakarmi J.J., Mandal P.K., and Chatterjee S. 2013. Effect of Organic Dyes on the Performance of $\mathrm{ZnO}$ Based Dye Sensitized Solar Cells. Applied Solar Energy, Springer 49(1): 40-45.

Mukode S. and Futata H. 1989. A semiconductive humidity sensor. Sensors and Actuators 16: $1-11$.

Pradhan B., Kumar S., Amal B., and. Pal B. 2007. Vertically aligned $\mathrm{ZnO}$ Nanowire Arrays in Rose Bengal based Dye Sensitized Solar Cells. Solar Energy Materials and Solar Cells 91: 769-773.

Shimizu Y., Shimabukuro M., Arai H. and Seiyama T. 1989. Humidity sensitive characteristics of $\mathrm{La}^{3+}$-doped and un doped $\mathrm{SrSnO}_{3}$. Journal of Electrochemical Society 136: 1206-1210.

Srivastava A., Jain K., Rashmi, Srivastava A. K. and Lakshmikumar S.T. 2006. Materials Chemistry and Physics 97: 85-91.

Sun A., Huang L., Li Y. 2009. Study on humidity sensing property based on $\mathrm{TiO}_{2}$ porous film and polystyrene sulfonic sodium. Sensors and actuators B 139(2): 543-547.

Yamazoe, N., Fuchigami J., Kishikawa M., and Seiyama T. 1979. Interactions of tin oxide surface with $\mathrm{O}_{2}, \mathrm{H}_{2} \mathrm{O}$ and $\mathrm{H}_{2}$. Surface Science 86: 335-344. 